\title{
Collision-Induced Effects on the Dielectric Properties of Liquid Dimethylsulfoxide
}

Sérgio M. Vechi and Munir S. Skaf

2002, vol 13, No 5, 583-591

The equations (1), (2), (5), (21), (23) and (24) were misprinted. The full correct equations are given as follows:

$v(i, j)=\sum_{\alpha \gamma}\left[v_{\alpha \gamma}^{l j}\left(\left|\mathbf{r}_{\alpha}^{i}-\mathbf{r}_{\gamma}^{j}\right|\right)+v_{\alpha \gamma}^{c o u l}\left(\left|\mathbf{r}_{\alpha}^{i}-\mathbf{r}_{\gamma}^{j}\right|\right)\right]$

$$
\frac{\left[\varepsilon(0)-\varepsilon_{\infty}\right]\left[\varepsilon_{\infty}+2 \varepsilon(0)\right]}{\varepsilon(0)}=\frac{N \mu^{2}}{V k_{B} T \varepsilon_{0}} L g_{K}
$$

$v_{\alpha \gamma}^{l j}=4 \varepsilon_{\alpha \gamma}\left[\left(\frac{\sigma_{\alpha \gamma}}{r}\right)^{12}-\left(\frac{\sigma_{\alpha \gamma}}{r}\right)^{6}\right]$

(2)

$$
\alpha(\omega)=\frac{\left[\varepsilon(0)-\varepsilon_{\infty}\right] \omega}{n(\omega) c} \varepsilon^{\prime \prime}(\omega)
$$

$$
\alpha=\left[\begin{array}{lll}
7.601 & 0.705 & 0.000 \\
0.705 & 7.006 & 0.000 \\
0.000 & 0.000 & 8.676
\end{array}\right] \AA^{3}
$$

$$
F(\omega)=\frac{\alpha(\omega) n(\omega)}{\varepsilon(0)-\varepsilon_{\infty}}=\omega^{2} \int_{0}^{\infty} \Phi(t) \cos (\omega) d t
$$

